\title{
$t \boldsymbol{J}$-model in terms of equations with variational derivatives
}

\author{
Yu.A.Izyumov, N.I.Chashchin \\ Institute for Metal Physics of the Russian Academy of Sciences, \\ Ural Division, 18 S.Kovalevskaya St., 620219 Ekaterinburg, Russia
}

Received June 17, 1998

For a $t J$-model in the $X$-operators representation a generating functional of the field describing fluctuations of matrix elements of electron hopping on a lattice is presented. The first order functional derivative with respect to this field determines the electron Green function, while the second order derivatives determine the boson Green functions of collective excitations in the system. Thus, the Kadanoff-Baym approach in the theory of fermi system with a weak Coulomb interaction is generalized on the opposite limit of systems with strong correlations. A chain of equations for different order variational derivatives were obtained, and a method was suggested based on iterations over the parameters of a $t J$-model: the hopping matrix element and the exchange integral. This approach corresponds to a self-consistent Born approximation, not for the effective but for the original Hamiltonian. A scheme of calculation of the dynamical spin susceptibility is analyzed with self-consistent corrections of the first and second order. Connection of this approach with the diagram technique for $X$-operators is discussed.

Key words: $t J$-model, Hubbard operators, Green's functions, antiferromagnetism

PACS: $71.27 .+a, 74.90 .+n$

\section{Introduction}

A $t J$-model is the basic working model in the theory of strongly correlated electron systems. It is convenient for the study of an interaction of charge and spin degrees of freedom, because the model describes the correlated motion of electrons on the lattice. The model is given by the Hamiltonian (see [1]):

$$
H=\sum_{i j \sigma} t_{i j}\left(1-n_{i \bar{\sigma}}\right) C_{i \sigma}^{\dagger} C_{j \sigma}\left(1-n_{j \bar{\sigma}}\right)+\sum_{i j} J_{i j}\left(\mathbf{S}_{i} \mathbf{S}_{j}-\frac{1}{4} n_{i} n_{j}\right) .
$$

Here $C_{i \sigma}\left(C_{i \sigma}^{\dagger}\right)$ is an operator of annihilation (creation) of an electron on site $i$ with spin $\sigma, \mathbf{S}_{i}$ - operator of spin, and $n_{i}$ - operator of the electron number on a 
site. Hopping matrix elements $t_{i j}$ and exchange integrals $J_{i j}$ are usually taken in the nearest neighbours approximations, so the model contains only two energy parameters: $t$ and $J$.

At half-filling, when the number of electrons on a site is $n=1$, the model (1.1) reduces to the Heisenberg model and has a dielectric antiferromagnetic ground state. When a deviation from half-filling takes place then some concentration of holes $\delta=1-n$ appears. They strongly interact with the magnetic order and locally deform it. As a result, a compound quasiparticle (magnetic polaron) appears, being a carrier of an electron charge in the system. A mathematical description of this situation is achieved by the transformation of Hamiltonian (1.1) into an effective Hamiltonian of a hole-magnon interaction. The self-consistent Born approximation (SCBA) is an approximation leading to a magnetic polaron picture inside the antiferromagnetic phase. The last one exists when $\delta<\delta_{\mathrm{c}}$, where critical concentration $\delta_{\mathrm{c}} \ll 1[1]$. It is well known that in high- $T_{\mathrm{c}}$-compounds the superconducting state appears outside the antiferromagnetic phase when $\delta>\delta_{\mathrm{c}}$, therefore, the question about applicability of the magnetic polaron picture for this concentration region is left open. For this reason a lot of new attempts are made to study the properties of the model (1.1) at $\delta>\delta_{\mathrm{c}}[1]$.

We find rather perspective here an approach on the basis of equations with variational derivatives in the spirit of the Kadanoff-Baym scheme [2]. At the beginning it was applied to the description of usual Fermi systems with a weak Coulomb interaction. Our aim is to develop a similar approach for the opposite case of systems with a strong Coulomb interaction, for example, for Hamiltonian (1.1) describing the correlated motion of electrons. The most convenient way to do that is to use the Hamiltonian of the $t J$-model in terms of the Hubbard $X$-operators. Then, by the analogy with usual Fermi operators, one can introduce fluctuating fields, corresponding to the hopping term in the Hamiltonian, in contrast to Kadanoff-Baym who introduced fluctuating fields of the potential interaction. As the result of such an approach, equations for generating functional $\mathcal{Z}$ and variational derivatives of $\mathcal{Z}$ over these fields are derived. These equations are convenient for iterations with respect to parameters $t$ and $J$, in contrast to Kadanoff-Baym equations convenient for iterations with respect to Coulomb interaction $U$.

The obtained equations allow one to develop a scheme of the self-consistent Born approximation (SCBA), not for the effective Hamiltonian, adopted for the description of the antiferromagnetic phase, but for the original Hamiltonian (1.1) and for the paramagnetic state as a ground state of the system.

The first attempt of such an approach was given by us in [3].

\section{Generating potential in terms of $X$-operators}

Because of the projection factors $\left(1-n_{i \sigma}\right)$ in the hopping term of the Hamiltonian, it is convenient to rewrite it in terms of $X$-operators:

$$
X_{i}^{\sigma 0}=\left(1-n_{i \bar{\sigma}}\right) C_{i \sigma}^{\dagger}, \quad X_{i}^{\sigma \sigma}=C_{i \sigma}^{\dagger} C_{i \sigma}, \quad X_{i}^{\sigma \bar{\sigma}}=C_{i \sigma}^{\dagger} C_{i \bar{\sigma}}
$$


Here the first $X$-operator is Fermi-like (f-type), and the others are Bose-like (btype). One can get then [4]

$$
H=\sum_{i \sigma} \varepsilon_{\sigma} X_{i}^{\sigma \sigma}+\sum_{i j \sigma} t_{i j}^{\sigma} X_{i}^{\sigma 0} X_{j}^{0 \sigma}+\frac{1}{2} \sum_{i j \sigma} J_{i j}\left(X_{i}^{\bar{\sigma} \sigma} X_{j}^{\sigma \bar{\sigma}}-X_{i}^{\sigma \sigma} X_{j}^{\bar{\sigma} \bar{\sigma}}\right) .
$$

We included external magnetic field $h$, that is why term $\varepsilon_{\sigma}=-\sigma h / 2$ appeared. We also introduced in a formal way hopping matrix element $t_{i j}$ by spin index $\sigma$.

We shall denote by figures complex indices including site $i$ and imaginary time $\tau$, so that $1=(i \tau)$, etc. Equations of motion for $\mathrm{f}$ - and b-operators are written in the form:

$$
\begin{array}{r}
-\frac{\mathrm{d} X_{1}^{0 \sigma}}{\mathrm{d} \tau_{1}}=\left[X_{1}^{0 \sigma}, H-\mu N\right]=\left(\varepsilon_{\sigma}-\mu\right) X_{1}^{0 \sigma}+\left(1-n_{i \bar{\sigma}}\right) t_{11^{\prime}}^{\sigma} X_{1^{\prime}}^{0 \sigma}+ \\
+X_{1}^{\bar{\sigma} \sigma} t_{11^{\prime}}^{\bar{\sigma}} X_{1^{\prime}}^{0 \bar{\sigma}}+J_{11^{\prime}}\left(X_{1}^{0 \bar{\sigma}} X_{1^{\prime}}^{\bar{\sigma} \sigma}-X_{1}^{\sigma \sigma} X_{1^{\prime}}^{\bar{\sigma} \bar{\sigma}}\right), \\
-\frac{\mathrm{d} X_{1}^{\sigma \bar{\sigma}}}{\mathrm{d} \tau_{1}}=\left[X_{1}^{\sigma \bar{\sigma}}, H-\mu N\right]=\left(\varepsilon_{\bar{\sigma}}-\varepsilon_{\sigma}\right) X_{1}^{\sigma \bar{\sigma}}-\left(X^{\sigma 0} t^{\sigma}\right)(1) X_{1}^{0 \bar{\sigma}}+ \\
+X_{1}^{\sigma 0}\left(t^{\bar{\sigma}} X^{0 \bar{\sigma}}\right)(1)+\left(X^{\sigma \bar{\sigma}} J\right)(1) \sigma m_{1}-X_{1}^{\sigma \bar{\sigma}}(J \sigma m)(1) .
\end{array}
$$

As usual, the summation (integrating) is implied over repeated indices because we introduced the quantities

$$
t_{11^{\prime}}=\delta\left(\tau-\tau^{\prime}\right) t_{i i^{\prime}}, \quad J_{11^{\prime}}=\delta\left(\tau-\tau^{\prime}\right) J_{i i^{\prime}} .
$$

Here and further we use a notation for matrix products, for example,

$$
\left(t X^{0 \sigma}\right)(1) \equiv t_{11^{\prime}} X_{1^{\prime}}^{0 \sigma}, \ldots
$$

Finally, by $n_{i \sigma}$ and $m_{i}$ we denote the number of electrons on a site and local magnetization

$$
n_{1 \sigma}=X_{1}^{\sigma \sigma}, \quad \sigma m_{1}=n_{1 \sigma}-n_{1 \bar{\sigma}} .
$$

Instead of quantities $n_{1 \sigma}$ it is convenient to use $F_{1}^{\sigma}=1-n_{1 \bar{\sigma}}$, being an anticommutator of two f-operators

$$
\left[X_{1}^{0 \sigma}, X_{1}^{\sigma 0}\right]_{+}=F_{1}^{\sigma}
$$

Now we introduce the generating potential

$$
\begin{gathered}
\mathcal{Z}\left[t^{\sigma}\right]=\operatorname{Sp}\left\{\mathrm{e}^{-\beta(H-\mu N)} \hat{\mathrm{T}} \mathrm{e}^{-\mathcal{F}\left[t^{\sigma}\right]}\right\}, \\
\mathcal{F}\left[t^{\sigma}\right]=\sum_{\sigma} X_{1^{\prime}}^{\sigma 0} t_{1^{\prime} 2^{\prime}}^{\sigma} X_{2^{\prime}}^{0 \sigma},
\end{gathered}
$$

where $\eta_{1^{\prime} 2^{\prime}}^{\sigma}$ is an auxiliary external field depending on thermodynamic time and spin. Here $\beta=1 / T$ and $\hat{\mathrm{T}}$ is a time-ordering operator. Since $\mathcal{F}\left[\eta^{\sigma}\right]$ coincides in 
the form with the hopping term in the Hamiltonian, the quantity $t_{1^{\prime} 2^{\prime}}^{\sigma}$ is to be considered as a fluctuating field of the electron hopping on the lattice.

The quantity $\mathcal{Z}\left[t^{\sigma}\right]$ is useful for determining the Green functions of the system, as its variational derivatives over $t_{12}^{\sigma}$

$$
\begin{aligned}
& \mathcal{G}_{\sigma}(12)=-\left\langle\hat{\mathrm{T}} X_{1}^{0 \sigma} X_{2}^{\sigma 0}\right\rangle=-\frac{1}{\mathcal{Z}} \frac{\delta \mathcal{Z}}{\delta t_{12}^{\sigma}}, \\
& \mathcal{D}_{1}^{\sigma}(12)=\left\langle\hat{\mathrm{T}} X_{1}^{\sigma \bar{\sigma}} X_{2}^{\bar{\sigma} \sigma}\right\rangle=-\frac{1}{\mathcal{Z}} \frac{\delta^{2} \mathcal{Z}}{\delta t_{12}^{\bar{\sigma}} \delta t_{21}^{\sigma}} \\
& \mathcal{D}_{0}^{\sigma \sigma^{\prime}}(12)=\left\langle\hat{\mathrm{T}} n_{1 \sigma} n_{2 \sigma^{\prime}}\right\rangle=\frac{1}{\mathcal{Z}} \frac{\delta^{2} \mathcal{Z}}{\delta t_{11}^{\sigma} \delta t_{22}^{\sigma^{\prime}}}
\end{aligned}
$$

It is clear from these definitions that $\mathcal{G}_{\sigma}$ is the Green function of itinerant electrons, and two others are the Green functions of collective Bose-like excitations: magnons and plasmons. The Bose-like Green functions are actually two-particle functions because b-operator may be presented as a product of f-operators: $X_{1}^{\sigma \bar{\sigma}}=$ $X_{1}^{\sigma 0} X_{1}^{0 \bar{\sigma}}, \quad n_{1 \sigma}=X_{1}^{\sigma 0} X_{1}^{0 \sigma}$.

For the generating functional one can derive the equation of motion with variational derivatives:

$$
K_{\sigma}\left(11^{\prime}\right) \frac{\delta \mathcal{Z}}{\delta t_{1^{\prime} 2}^{\sigma}}=-\delta_{12}\left\langle F_{1}^{\sigma}\right\rangle \mathcal{Z}+\gamma_{\sigma}\left(1 ; 1^{\prime} 3^{\prime}\right) \frac{\delta^{2} \mathcal{Z}}{\delta t_{1^{\prime} 2}^{\sigma} \delta t_{3^{\prime} 3^{\prime}}^{\bar{\sigma}}}+\gamma_{\bar{\sigma}}\left(1 ; 1^{\prime} 3^{\prime}\right) \frac{\delta^{2} \mathcal{Z}}{\delta t_{3^{\prime} 2}^{\sigma} \delta t_{1^{\prime} 3^{\prime}}^{\bar{\sigma}}}
$$

or in the conjugated form

$$
\frac{\delta \mathcal{Z}}{\delta t_{12^{\prime}}^{\sigma}} K_{\sigma}\left(2^{\prime} 2\right)=-\delta_{12}\left\langle F_{1}^{\sigma}\right\rangle \mathcal{Z}+\frac{\delta^{2} \mathcal{Z}}{\delta t_{12^{\prime}}^{\sigma} \delta t_{3^{\prime} 3^{\prime}}^{\bar{\sigma}}} \widetilde{\gamma}_{\sigma}\left(3^{\prime} 2^{\prime} ; 2\right)+\frac{\delta^{2} \mathcal{Z}}{\delta t_{13^{\prime}}^{\sigma} \delta t_{3^{\prime} 2^{\prime}}^{\bar{\sigma}}} \widetilde{\gamma}_{\bar{\sigma}}\left(3^{\prime} 2^{\prime} ; 2\right) .
$$

Here $K_{\sigma}$ is a differential operator, $\gamma_{\sigma}$ and $\widetilde{\gamma}_{\sigma}$ are matrix elements of the Hamiltonian.

$$
\left.\begin{array}{c}
K_{\sigma}(12)=-\left(\frac{\partial}{\partial \tau_{1}}+\varepsilon_{\sigma}\right) \delta(1-2)-t_{12}^{\sigma} \\
\gamma_{\sigma}(1 ; 23)=t_{12}^{\sigma} \delta_{13}+\delta_{12} J_{13} \\
\widetilde{\gamma}_{\sigma}(32 ; 1)=\delta_{31} t_{21}^{\sigma}+J_{31} \delta_{21}
\end{array}\right\} .
$$

One must add the obvious relation to equations (2.11) and (2.12):

$$
\left\langle F_{1}^{\sigma}\right\rangle=1+\frac{1}{\mathcal{Z}} \frac{\delta \mathcal{Z}}{\delta t_{11}^{\bar{\sigma}}} .
$$

Equation (2.11) is the basic equation corresponding to the Kadanoff-Baym method applied to a strongly correlated system. In contrast to the theory of a usual Fermi-system here variational derivatives are taken over the fluctuating field of the hopping term but not over the potential one. For this reason equation (2.11) is convenient for iterations with respect to parameters $t$ and $J$, while in the usual 
Fermi-system variational derivatives are taken over the parameters of an electron interaction.

The basic equation (2.11) connects the first derivative of $\mathcal{Z}$ with the second one. To find an equation for the second derivative it is necessary to take a variational derivative of equation (2.11) over $t_{34}^{\bar{\sigma}}$. Then one obtains an equation connecting the second derivative with the third one:

$$
\begin{aligned}
K_{\sigma}\left(11^{\prime}\right) & \frac{\delta^{2} \mathcal{Z}}{\delta t_{1^{\prime} 2}^{\sigma} \delta t_{34}^{\bar{\sigma}}}=-\delta_{12}\left(\frac{\delta \mathcal{Z}}{\delta t_{34}^{\bar{\sigma}}}+\frac{\delta^{2} \mathcal{Z}}{\delta t_{11}^{\bar{\sigma}} \delta t_{34}^{\bar{\sigma}}}\right)+\delta_{14} \frac{\delta^{2} \mathcal{Z}}{\delta t_{12}^{\sigma} \delta t_{31}^{\bar{\sigma}}}+ \\
& +\gamma_{\sigma}\left(1 ; 1^{\prime} 3^{\prime}\right) \frac{\delta^{3} \mathcal{Z}}{\delta t_{1^{\prime} 2}^{\sigma} \delta t_{3^{\prime} 3^{\prime}}^{\bar{\sigma}} \delta t_{34}^{\bar{\sigma}}}+\gamma_{\bar{\sigma}}\left(1 ; 1^{\prime} 3^{\prime}\right) \frac{\delta^{3} \mathcal{Z}}{\delta t_{3^{\prime} 2}^{\sigma} \delta t_{1^{\prime} 3^{\prime}}^{\bar{\sigma}} \delta t_{34}^{\bar{\sigma}}} .
\end{aligned}
$$

We write the following equation of the infinite chain obtained by the differentiation of the previous equation with respect to $t_{56}^{\bar{\sigma}}$ :

$$
\begin{array}{r}
K_{\sigma}\left(11^{\prime}\right) \frac{\delta^{3} \mathcal{Z}}{\delta t_{1^{\prime} 2}^{\sigma} \delta t_{34}^{\bar{\sigma}} \delta t_{56}^{\bar{\sigma}}}=-\delta_{12}\left(\frac{\delta^{2} \mathcal{Z}}{\delta t_{34}^{\bar{\sigma}} \delta t_{56}^{\bar{\sigma}}}+\frac{\delta^{3} \mathcal{Z}}{\delta t_{11}^{\bar{\sigma}} \delta t_{34}^{\bar{\sigma}} \delta t_{56}^{\bar{\sigma}}}\right)+ \\
+\delta_{14} \frac{\delta^{3} \mathcal{Z}}{\delta t_{12}^{\sigma} \delta t_{34}^{\bar{\sigma}} \delta t_{56}^{\bar{\sigma}}}+\delta_{16} \frac{\delta^{3} \mathcal{Z}}{\delta t_{12}^{\sigma} \delta t_{34}^{\bar{\sigma}} \delta t_{56}^{\bar{\sigma}}}+ \\
+\gamma_{\sigma}\left(1 ; 1^{\prime} 3^{\prime}\right) \frac{\delta^{4} \mathcal{Z}}{\delta t_{1^{\prime} 2^{\sigma}}^{\sigma} \delta t_{3^{\prime} 3^{\prime}}^{\bar{\sigma}} \delta t_{34}^{\bar{\sigma}} \delta t_{56}^{\bar{\sigma}}}+\gamma_{\bar{\sigma}}\left(1 ; 1^{\prime} 3^{\prime}\right) \frac{\delta^{4} \mathcal{Z}}{\delta t_{3^{\prime} 2}^{\sigma} \delta t_{1^{\prime} 3^{\prime}}^{\bar{\sigma}} \delta t_{34}^{\bar{\sigma}} \delta t_{56}^{\bar{\sigma}}} .
\end{array}
$$

After differentiation in these equations one has to put variable $t_{12}^{\sigma}$ equal to matrix element $t_{12}$ standing in the hopping term of the Hamiltonian.

In figures 1 and 2 a graph representation of the basic equation is given. A four-

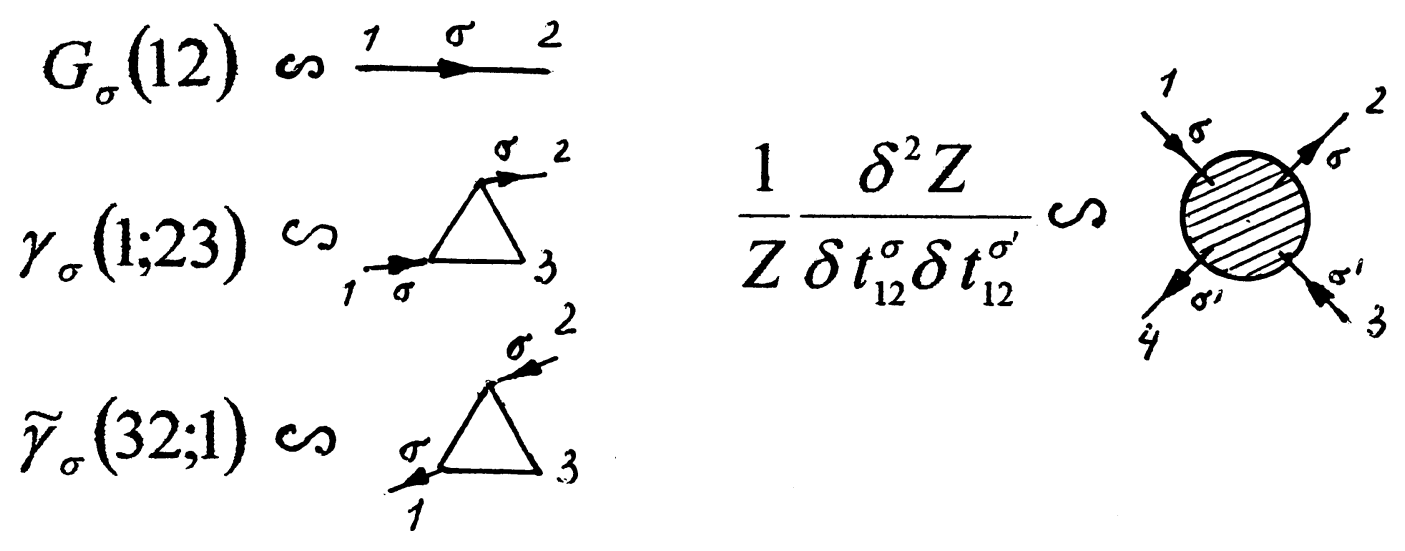

Figure 1. Graphic elements.

tail diagram denoting a second order variational derivative is not a vertex part but a two-particle Green function. We shall see later that the zero approximation over $\gamma_{\sigma}$ contains a nonconnected part, generating in the equation the first order graphs, corresponding in the usual Fermi system theory to Hartree-Fock terms. The connected part of the zero approximation generates new type graphs absent in the Fermi system theory. Corrections of the first and next orders generate vertex parts, more complicated in comparison with the usual Fermi systems $[5,6]$. 


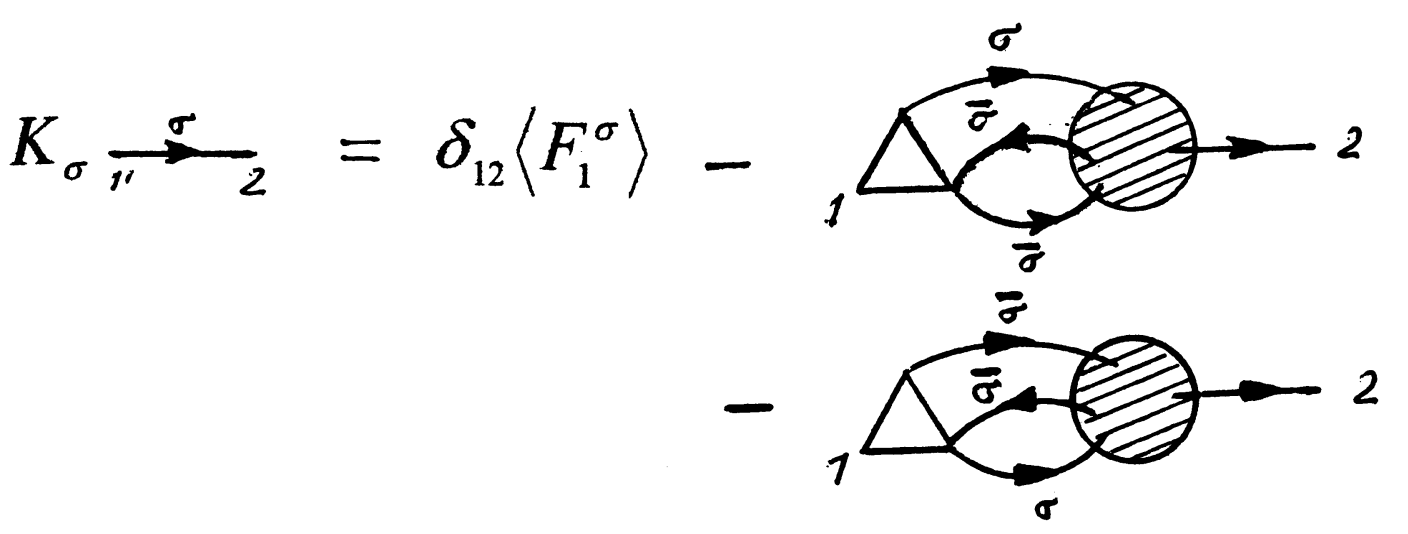

Figure 2. Graphic representation of the basic equation (2.11).

\section{Calculation of variational derivatives}

In accordance with the graph representation for the second derivative shown in figure 1, we shall look for the form:

$$
\frac{1}{\mathcal{Z}} \frac{\delta^{2} \mathcal{Z}}{\delta t_{12}^{\sigma} \delta t_{34}^{\bar{\sigma}}}=\frac{\delta \Phi}{\delta t_{11^{\prime}}^{\sigma}} \frac{\delta \Phi}{\delta t_{33^{\prime}}^{\bar{\sigma}}} \hat{\Gamma}_{2}\left(\sigma 1^{\prime} 2^{\prime} ; \bar{\sigma} 3^{\prime} 4^{\prime}\right) \frac{\delta \Phi}{\delta t_{2^{\prime} 2}^{\sigma}} \frac{\delta \Phi}{\delta t_{4^{\prime} 4}^{\bar{\sigma}}} .
$$

The quantity $\delta \Phi / \delta t$ is an electron Green function $\left(\mathcal{Z}=\mathrm{e}^{\mathrm{i} \phi}\right)$, while $\hat{\Gamma}_{2}$ is to be considered a vertex part. In the theory of the usual Fermi system $\hat{\Gamma}_{2}$ should be a real vertex part of an electron-electron interaction. In the case of strongly correlated electrons the situation is different and in representation (3.1) $\hat{\Gamma}_{2}$ should be an operator. It acts on the Green function standing by the left- and the righthand side and can transform them into other Green functions.

The representation (3.1) is, however, convenient because it opens a way to calculate the second and higher derivatives. For this purpose equation (3.1) has to be multiplied by the left- and the right-hand side operator quantities (2.13). Then it is written:

$$
\begin{array}{r}
K_{\sigma}\left(11^{\prime}\right) K_{\bar{\sigma}}\left(33^{\prime}\right) \frac{1}{\mathcal{Z}} \frac{\delta^{2} \mathcal{Z}}{\delta t_{1^{\prime} 2^{\prime}}^{\sigma} \delta t_{3^{\prime} 4^{\prime}}^{\bar{\sigma}}} K_{\sigma}\left(2^{\prime} 2\right) K_{\bar{\sigma}}\left(4^{\prime} 4\right)= \\
\left(K_{\sigma} \frac{\delta \Phi}{\delta t^{\sigma}}\right)\left(11^{\prime}\right)\left(K_{\bar{\sigma}} \frac{\delta \Phi}{\delta t^{\sigma}}\right)\left(33^{\prime}\right) \hat{\Gamma}_{2}\left(\sigma 1^{\prime} 2^{\prime} ; \bar{\sigma} 3^{\prime} 4^{\prime}\right)\left(\frac{\delta \Phi}{\delta t^{\sigma}} K_{\sigma}\right)\left(2^{\prime} 2\right)\left(\frac{\delta \Phi}{\delta t^{\bar{\sigma}}} K_{\bar{\sigma}}\right)\left(4^{\prime} 4\right) .
\end{array}
$$

In the right-hand side of this relation we may use equations (2.11) and (2.12), and in the left-hand side - equation (2.16) (and a conjugated one). It allows one to find $\hat{\Gamma}_{2}$ as a series in powers of $\gamma_{\sigma}$ and $\gamma_{\bar{\sigma}}$. After rather cumbersome calculations we find quantities $\hat{\Gamma}_{2}$ for two second variation derivatives with equal and opposite spin indices. In the zero approximation

$$
\hat{\Gamma}_{2}^{0}(\sigma 12 ; \sigma 34)=\frac{K_{\sigma}^{0}(12) K_{\sigma}^{0}(34)-K_{\sigma}^{0}(14) K_{\sigma}^{0}(32)}{\left\langle F_{1}^{\sigma}\right\rangle\left\langle F_{2}^{\sigma}\right\rangle\left\langle F_{3}^{\sigma}\right\rangle\left\langle F_{4}^{\sigma}\right\rangle}\left[d_{0}^{\bar{\sigma} \bar{\sigma}}(13)+\left\langle F_{1}^{\sigma}\right\rangle\left\langle F_{3}^{\sigma}\right\rangle\right]
$$




$$
\begin{aligned}
\hat{\Gamma}_{2}^{0}(\sigma 12 ; \bar{\sigma} 34) & =\delta_{13} \delta_{34} \frac{K_{\sigma}^{0}(12)}{\left\langle F_{1}^{\sigma}\right\rangle\left\langle F_{2}^{\sigma}\right\rangle\left\langle F_{1}^{\bar{\sigma}}\right\rangle}+\delta_{12} \delta_{23} \frac{K_{\bar{\sigma}}^{0}(34)}{\left\langle F_{4}^{\sigma}\right\rangle\left\langle F_{3}^{\bar{\sigma}}\right\rangle\left\langle F_{4}^{\bar{\sigma}}\right\rangle}+ \\
& +\frac{K_{\bar{\sigma}}^{0}(14) K_{\sigma}^{0}(32) \mathcal{D}_{1}^{\sigma}(31)}{\left\langle F_{1}^{\sigma}\right\rangle\left\langle F_{2}^{\sigma}\right\rangle\left\langle F_{3}^{\bar{\sigma}}\right\rangle\left\langle F_{4}^{\bar{\sigma}}\right\rangle}+\frac{K_{\sigma}^{0}(12) K_{\bar{\sigma}}^{0}(34)\left[d_{0}^{\bar{\sigma} \sigma}(13)+\left\langle F_{1}^{\sigma}\right\rangle\left\langle F_{3}^{\bar{\sigma}}\right\rangle\right]}{\left\langle F_{1}^{\sigma}\right\rangle\left\langle F_{2}^{\sigma}\right\rangle\left\langle F_{3}^{\bar{\sigma}}\right\rangle\left\langle F_{4}^{\bar{\sigma}}\right\rangle} .
\end{aligned}
$$

Both equations contain Bose-like Green functions for magnons and plasmons, however, now instead of quantities (2.10) we have

$$
d_{0}^{\sigma \sigma^{\prime}}(12)=\mathcal{D}_{0}^{\sigma \sigma^{\prime}}(12)-\left\langle F_{1}^{\sigma}\right\rangle\left\langle F_{2}^{\sigma^{\prime}}\right\rangle=\left\langle\hat{\mathrm{T}}\left[n_{1 \sigma}-\left\langle n_{1 \sigma}\right\rangle\right]\left[n_{2 \sigma^{\prime}}-\left\langle n_{2 \sigma^{\prime}}\right\rangle\right]\right\rangle .
$$

We see that expressions (3.3) and (3.4) contain operators $K_{\sigma}^{0}$ which act in relation (3.1) on electron Green functions. In this way the expression for the second derivatives of $\mathcal{Z}$ splits into a number of terms containing not four electron Green functions but a smaller number of them, three or two. We have used the fact that at the zero approximation, according to equation $(2.11), K_{\sigma}^{0}\left(\delta \Phi / \delta t^{\sigma}\right)$ is just $\left\langle F^{\sigma}\right\rangle$. As a result, the second derivative of $\mathcal{Z}$ can be written in a graph form (figures 3 and 4 ). When writing analytical expressions, one has to keep in mind the following
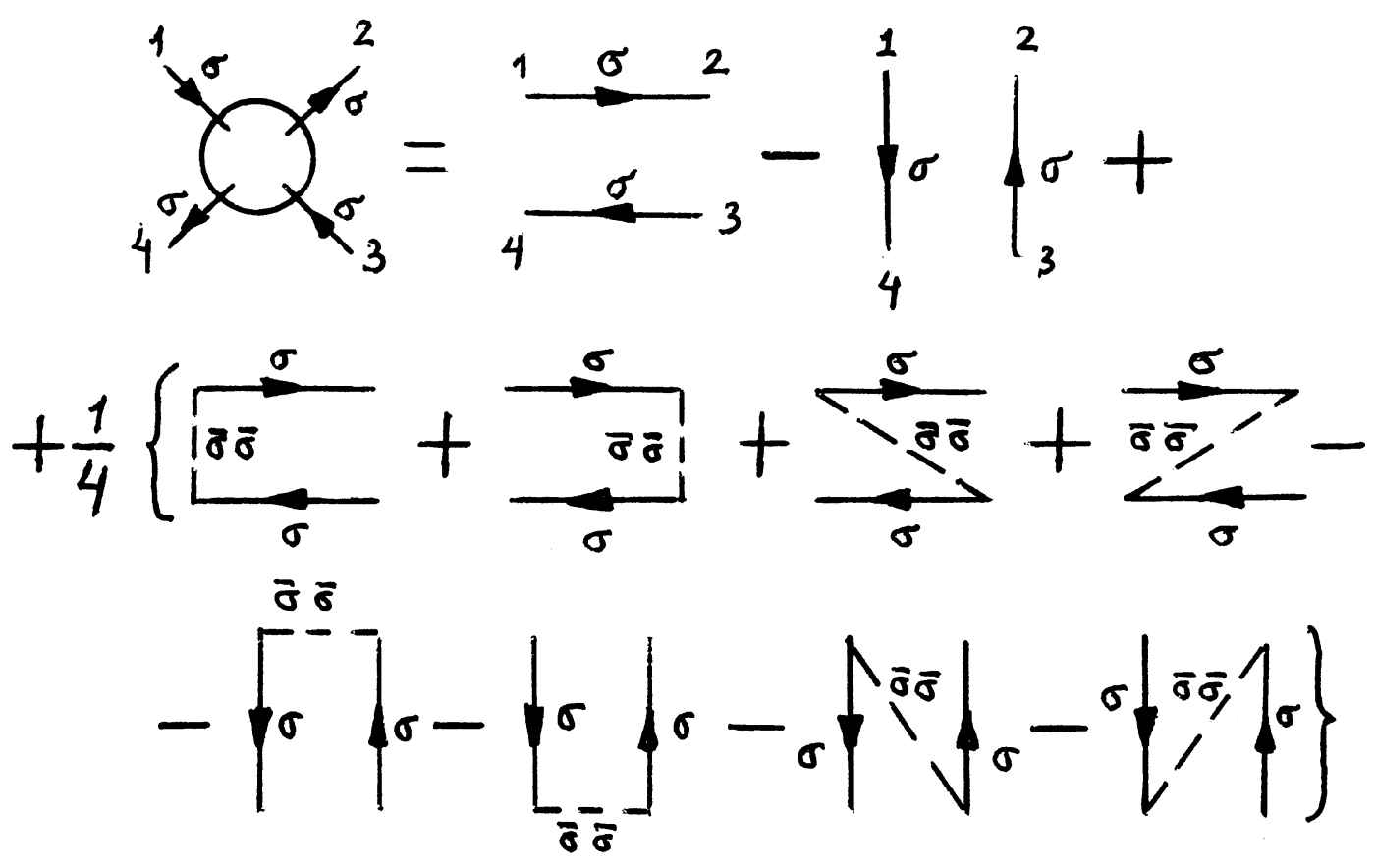

Figure 3. Graphic representation of $\frac{1}{\mathcal{Z}} \frac{\delta^{2} \mathcal{Z}}{\delta t_{12}^{\sigma} \delta t_{34}^{\sigma}}$ in zero approximation. Dasked lines denot plasmon Green functions (2.10).

rule: a complex vertex creates a numerical factor corresponding to each outgoing and ingoing electron line. For example, to the first connected graphs in figure 3 there corresponds factor $\left\langle F_{1}^{\sigma}\right\rangle^{-1}\left\langle F_{4}^{\sigma}\right\rangle^{-1}$. Coefficients $A^{\sigma}$ and $B^{\sigma}$ for the triple graphs in figure 4 are equal to

$$
A_{1}^{\sigma}=\left\langle F_{1}^{\sigma}\right\rangle+\left\langle F_{1}^{\bar{\sigma}}\right\rangle-\frac{1}{2} \sigma\left\langle m_{1}\right\rangle, \quad B_{2}^{\sigma}=\left\langle F_{2}^{\bar{\sigma}}\right\rangle-\left\langle F_{2}^{\sigma}\right\rangle+\frac{1}{2} \sigma\left\langle m_{1}\right\rangle .
$$


Besides, according to the mentioned rule, to these two graphs additional factors $\left\langle F^{\bar{\sigma}}\right\rangle^{-2}\left\langle F^{\sigma}\right\rangle^{-1}$ or $\left\langle F^{\sigma}\right\rangle^{-1}\left\langle F^{\sigma}\right\rangle^{-2}$ should be prescribed.
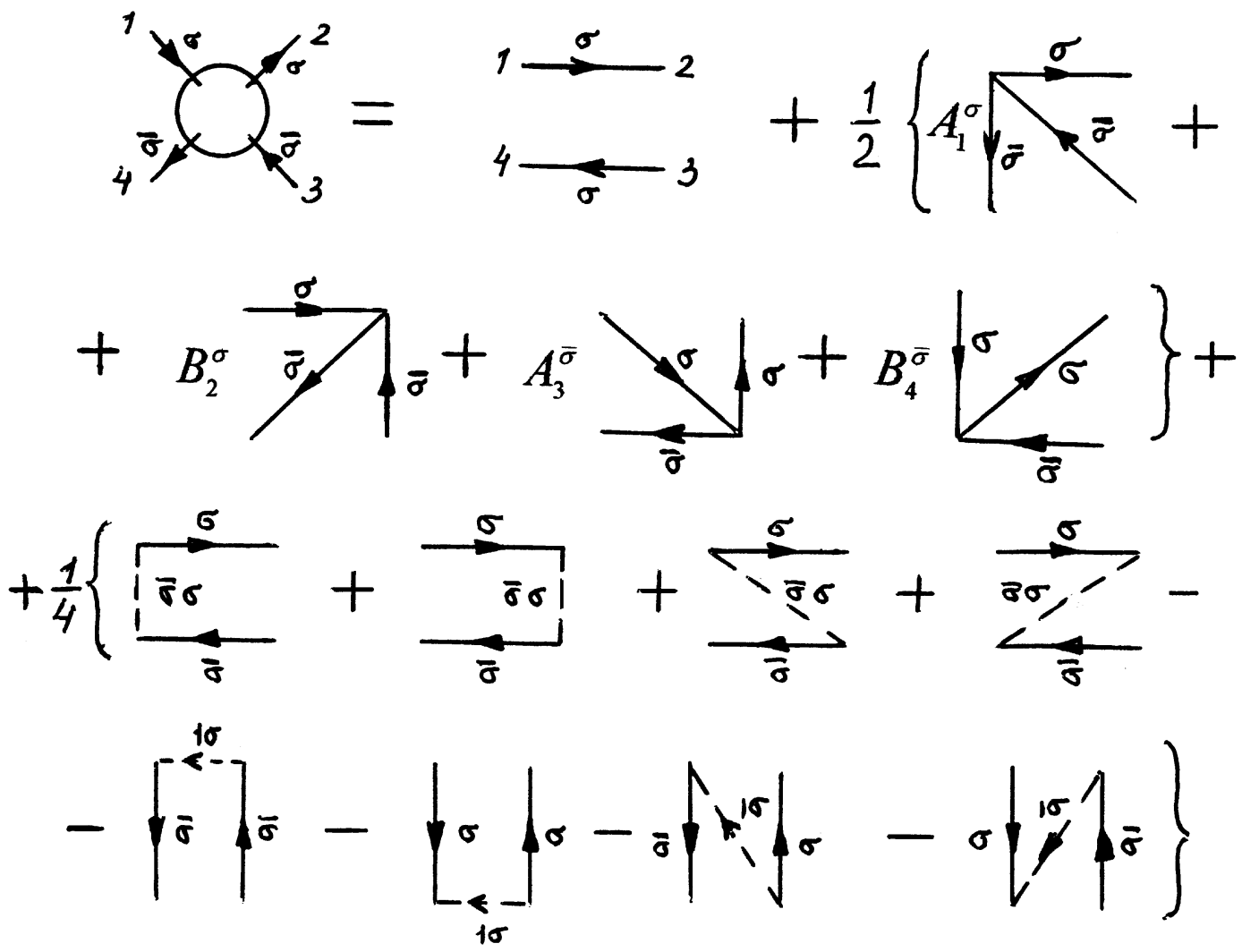

Figure 4. Graphic representation of $\frac{1}{\mathcal{Z}} \frac{\delta^{2} \mathcal{Z}}{\delta t_{12}^{\sigma} \delta t_{34}^{\bar{\sigma}}}$ in zero approximation. Dasked lines with an arrow denotes the magnon Green function (2.9).

The third order variational derivative is calculated in the same way. It is necessary to start from the relation of type (3.1) not with four but six factors $\delta \Phi / \delta t$. The complete expression is too complicated to write it down. It contains nonconnected diagrams corresponding to the product of the second derivative and the first one. Among the connected diagrams there are graphs constructed only from electron lines (five lines) and graphs with one boson line and four electron lines converging to one point (as in the pure electron graphs in figure 4). Finally, there are peculiar graphs containing a structure corresponding to a six-tailed one with pairs of joint electron lines (a flower with three clovers). Such type of graphs is shown in figure 5. One can check that a complete set of graphs for the third derivative provides the necessary symmetry arising from the possibility to change the order of differentiation. Besides, the symmetry is achieved according to the permutation of pair indices $(3,5)$ and $(4,6)$.

In principle, in the same way it would be possible to obtain a graphical representation for the fourth derivative (eight-tailed) of the zero approximation. 

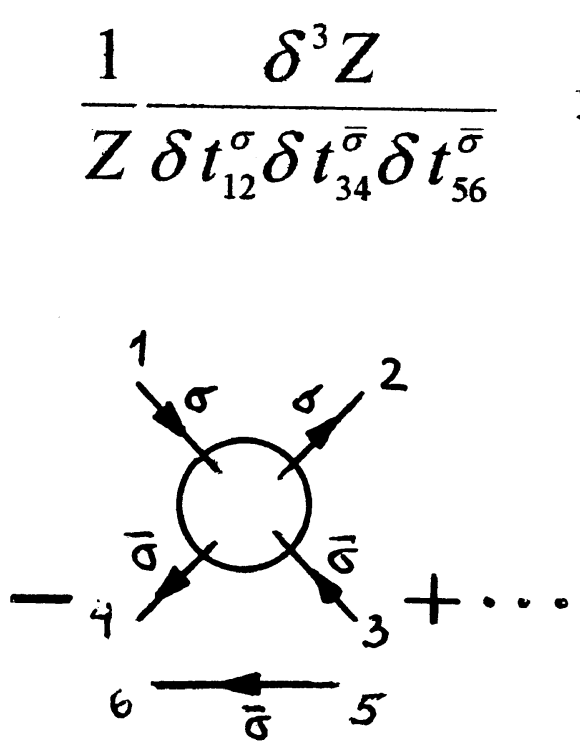
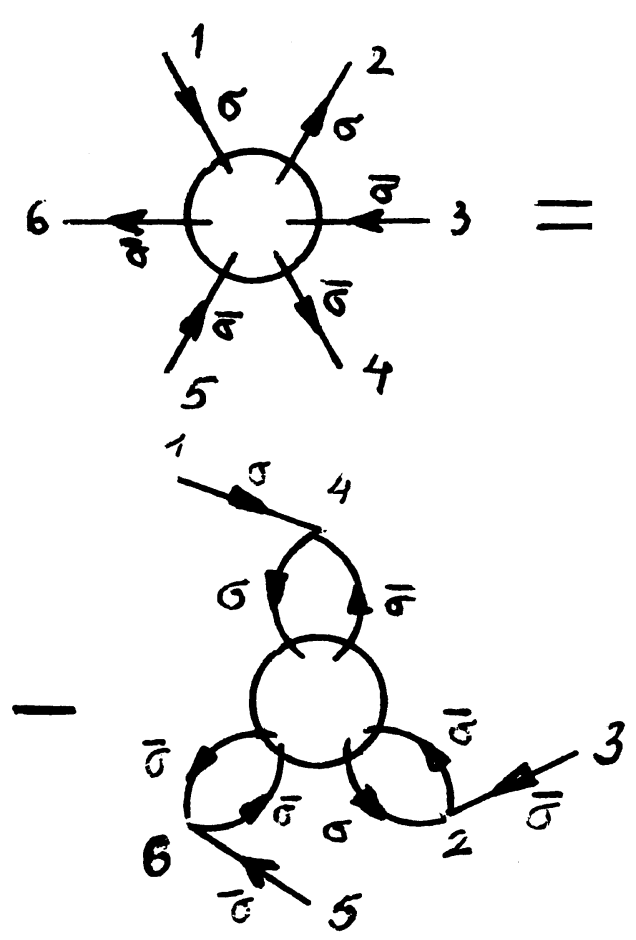

Figure 5. The third order variational derivative in zero approximation.

\section{The magnon Green function}

The magnon Green function can be found if the second derivative $\delta^{2} \mathcal{Z} / \delta t_{12}^{\bar{\sigma}} \delta t_{34}^{\sigma}$ is calculated in some approximation. According to definition (2.9), it is enough to equate indices $4=1$ and $3=2$. We calculate first $\mathcal{D}_{1}^{\sigma}(12)$ at the zero approximation. In the left- and the right-hand sides of the graph relation in figure 4 we make these indices equal to each other. Then, an equation for $\mathcal{D}_{1}^{\sigma}(12)$ appears, which we write in the analytical form:

$$
\begin{aligned}
& {\left[1-\left(\frac{1}{2} \sum_{\sigma_{1}} \frac{\left\langle n_{1}^{\sigma_{1}}\right\rangle}{\left\langle F_{1}^{\sigma_{1}}\right\rangle}\right)\left(\frac{1}{2} \sum_{\sigma_{2}} \frac{\left\langle n_{2}^{\sigma_{2}}\right\rangle}{\left\langle F_{2}^{\sigma_{2}}\right\rangle}\right)\right] \mathcal{D}_{1}^{\sigma}(12)=} \\
& -\mathcal{G}_{\bar{\sigma}}(12) \mathcal{G}_{\sigma}(21)\left[1+\frac{1}{2}\left(\frac{A_{1}^{\bar{\sigma}}\left\langle n_{1}^{\sigma}\right\rangle}{\left\langle F_{1}^{\bar{\sigma}}\right\rangle\left\langle F_{1}^{\sigma}\right\rangle^{2}}+\frac{A_{2}^{\sigma}\left\langle n_{2}^{\bar{\sigma}}\right\rangle}{\left\langle F_{2}^{\sigma}\right\rangle\left\langle F_{2}^{\bar{\sigma}}\right\rangle^{2}}+\frac{B_{1}^{\sigma}\left\langle n_{1}^{\bar{\sigma}}\right\rangle}{\left\langle F_{1}^{\sigma}\right\rangle\left\langle F_{1}^{\bar{\sigma}}\right\rangle^{2}}+\frac{B_{2}^{\bar{\sigma}}\left\langle n_{2}^{\sigma}\right\rangle}{\left\langle F_{2}^{\bar{\sigma}}\right\rangle\left\langle F_{2}^{\sigma}\right\rangle^{2}}\right)\right] \\
& -\frac{1}{4} \sum_{\sigma^{\prime}} \frac{1}{\left\langle F_{1}^{\sigma^{\prime}}\right\rangle\left\langle F_{2}^{\bar{\sigma}^{\prime}}\right\rangle} \mathcal{G}_{\bar{\sigma}}(12) d_{0}^{\bar{\sigma} \sigma}(21) \mathcal{G}_{\sigma}(21) .
\end{aligned}
$$

One can check here the rules of writing analytical expressions.

For the paramagnetic phase we have from here:

$$
\mathcal{D}_{1}^{\sigma}(12)=-\frac{1+n^{2} / 4}{1-n} \mathcal{G}(12) \mathcal{G}(21)
$$

We neglect the last term in expression (4.2) which changes only a numerical coefficient. If in this expression sites 1 and 2 are put to be the nearest neighbours 
and also time $\tau_{1}$ and $\tau_{2}$ to be equal, we obtain a relation between the spin and electron correlators coinciding with the one obtained in [4].

To include corrections of the first order in the magnon Green function we must calculate the second derivative with the accuracy up to the first order with respect to $t$ and $J$. Then, making the indices equal in the same way as in the zero approximation, we come to the equation, presented in figure 6. Its solution gives

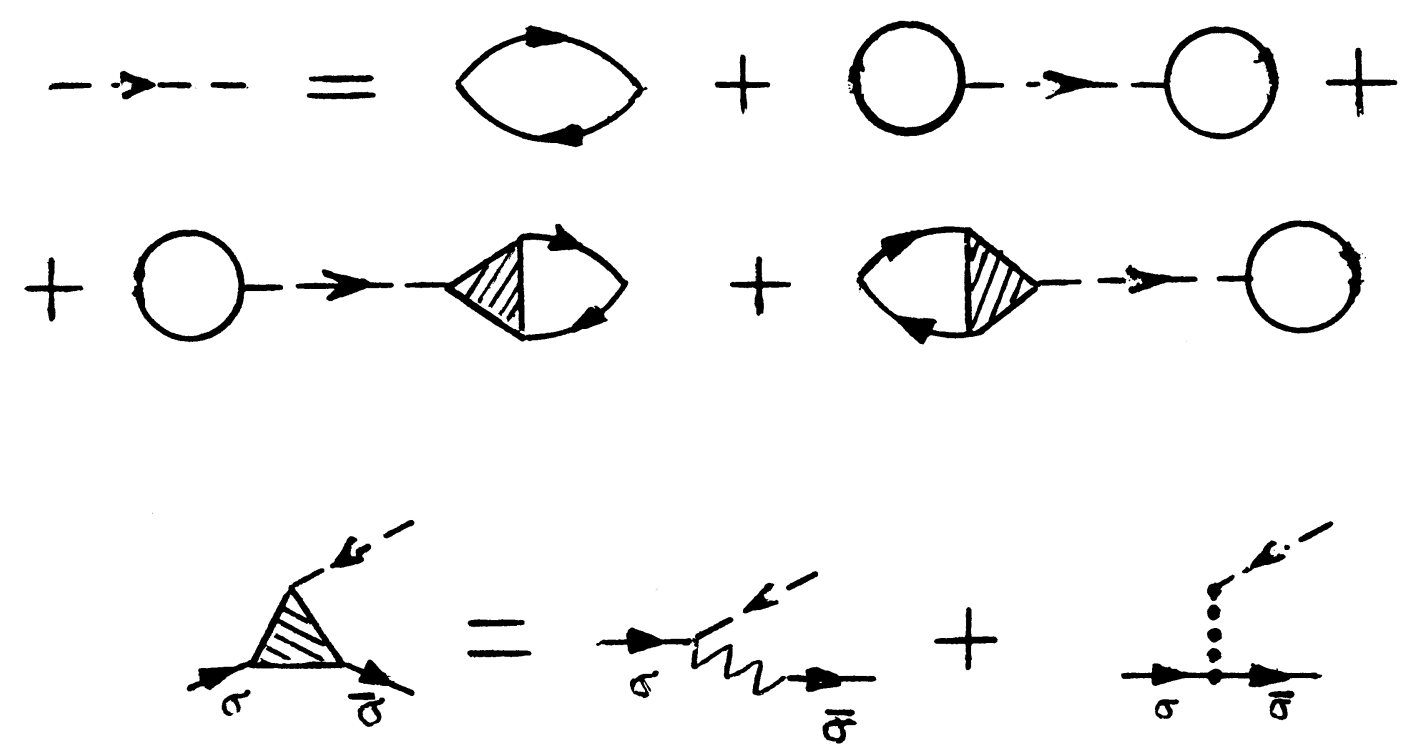

Figure 6. Equation for magnon Green function.

the magnon Green function of the form:

$$
\mathcal{D}_{1}^{\sigma}(\mathbf{q})=\frac{-\Pi(\mathbf{q})}{1-n-\frac{n}{2}[Q(\mathbf{q})+\Lambda(\mathbf{q})]-J(\mathbf{q}) \Pi(\mathbf{q})},
$$

where

$$
\{\Pi(\mathbf{q}), Q(\mathbf{q}), \Lambda(\mathbf{q})\}=\sum_{\mathbf{k}}\{1, \varepsilon(\mathbf{k}), \varepsilon(\mathbf{k}-\mathbf{q})\} \mathcal{G}(\mathbf{k}-\mathbf{q}) \mathcal{G}(\mathbf{k}) .
$$

corresponds to an electron loop and the loops with an inserted wavy line. Such expressions appeared earlier in the diagram technique with $X$-operators [7].

Result (4.3) is consistent with the expression obtained by us [3], with a difference in numerical coefficients. At $\mathbf{q}=\mathbf{0}$ the last term in the denominator can be neglected, thus, the ferromagnetic instability of the system is determined by the hopping term in the Hamiltonian. In contrast, at $\mathbf{q}=\mathbf{Q}=(\pi, \pi, \ldots) / a Q(\mathbf{q})+\Lambda(\mathbf{q})=$ 0 the antiferromagnetic instability is determined by the exchange term. In [3] we showed that in the Hubbard-1 approximation for electrons $\Pi(\mathbf{Q}, 0) \sim(1-n)$ and antiferromagnetic instability occurs only when $J \sim t$, which is not consistent with the known idea [1] that critical value $J / t$ for the appearance of antiferromagnetic order should be small, as half-filling $(1-n)$ is approached. It means that near $n=1$ for the analysis of antiferromagnetic instability it is probably necessary to 
go beyond the mean field approximation for electrons. The other possibility is to take into account the second order corrections for $t$ and $J$. In the next chapter we will give a preliminary analysis of these corrections without explicit calculations.

\section{On the second order corrections in the magnon Green func- tion}

It would be difficult to look for these corrections by the method of [3] because one should know self-energy of electrons up to the third order. In the present approach it is sufficient to have corrections up to only the second order for the second derivative. We need for this the forth order derivative (eight-tailed) in the zero approximation. As we saw earlier, the number of terms increases very fast with the order of the derivative, and a selection of actual diagrams is necessary. In our case (nearly half-filling) this selection might be done on the basis of parameter $1-n \ll 1$.

Let us look for the magnon Green function (4.2) of the zero approximation. Factor $1-n$ in the denominator does not yet mean a singularity because it may be cancelled by the numerator. However, it is necessary to pay attention to all the terms containing this factor in the denominator. Notice that this factor appeared due to the coefficient in the left-hand side of equation (4.1). We try to search for such factors in expressions for the third and fourth derivatives, however, we shall use now another method based on the Wick theorem for $X$-operators $[5,7]$.

We start from the second order derivative written in the form:

$$
\frac{1}{\mathcal{Z}} \frac{\delta^{2} \mathcal{Z}}{\delta t_{12}^{\sigma} \delta t_{34}^{\bar{\sigma}}}=\left\langle\hat{\mathrm{T}} \psi_{\sigma}(1) \psi_{\sigma}^{\dagger}(2) \psi_{\bar{\sigma}}(3) \psi_{\bar{\sigma}}^{\dagger}(4)\right\rangle
$$

where $\psi_{\sigma}(1) \equiv X_{1}^{0 \sigma}$ is a Fermi-like operator. When averaging in (4.4) with the Hamiltonian of the zero approximation, the average of T-product would be reduced to the pair-averages by the procedure based on the Wick theorem. We should take all the possible systems of pairing. First, consider the systems with the pairing of only $\psi$ and $\psi^{\dagger}$ operators, for example:

$$
\left\langle\hat{\mathrm{T}} \psi_{\sigma}(1) \psi_{\sigma}^{\dagger}(2) \psi_{\bar{\sigma}}(3) \psi_{\bar{\sigma}}^{\dagger}(4)\right\rangle=-G_{\bar{\sigma}}(32) G_{\sigma}(14)\left\langle\hat{\mathrm{T}} X_{2}^{\sigma \bar{\sigma}} X_{4}^{\bar{\sigma} \sigma}\right\rangle .
$$

Here $G_{\sigma}(12)$ is the fermion Green function of the zero approximation. Our approximation now is the one, in which we take only $\psi$-operators pairing and in expressions of type (5.2) the Green functions are replaced by the exact ones. They are related to the electron Green functions by the identity

$$
\mathcal{G}_{\sigma}(12)=G_{\sigma}(12)\left\langle F_{2}^{\sigma}\right\rangle \text {. }
$$

Taking into account in (5.1) only two systems of pairing, we can write

$$
\frac{1}{\mathcal{Z}} \frac{\delta^{2} \mathcal{Z}}{\delta t_{12}^{\sigma} \delta t_{34}^{\bar{\sigma}}} \approx\left[\mathcal{G}_{\sigma}(12) \mathcal{G}_{\bar{\sigma}}(34)\left\langle\hat{\mathrm{T}} F_{2}^{\sigma} F_{4}^{\bar{\sigma}}\right\rangle-\mathcal{G}_{\bar{\sigma}}(32) \mathcal{G}_{\sigma}(14)\left\langle\hat{\mathrm{T}} X_{2}^{\sigma \bar{\sigma}} X_{4}^{\bar{\sigma} \sigma}\right\rangle\right] \frac{1}{\left\langle F_{2}^{\sigma}\right\rangle\left\langle F_{4}^{\bar{\sigma}}\right\rangle}
$$


In this approximate relation we put indices $4=1$ and $3=2$, then we have an equation for the magnon Green function:

$$
\left[1-\frac{\left\langle n_{1}^{\sigma}\right\rangle}{\left\langle F_{1}^{\bar{\sigma}}\right\rangle} \frac{\left\langle n_{2}^{\bar{\sigma}}\right\rangle}{\left\langle F_{2}^{\sigma}\right\rangle}\right] \mathcal{D}_{1}^{\sigma}(12)=-\mathcal{G}_{\bar{\sigma}}(12) \mathcal{G}_{\sigma}(21) \frac{1+d_{0}^{\bar{\sigma} \sigma}(21)}{\left\langle F_{1}^{\bar{\sigma}}\right\rangle\left\langle F_{2}^{\sigma}\right\rangle} .
$$

Here we use the value for the electron Green function with coincided arguments: $\mathcal{G}_{\sigma}(11)=\left\langle X_{1}^{\sigma \sigma}\right\rangle=\left\langle n_{1}^{\sigma}\right\rangle$.

For the paramagnetic phase the coefficient in the left-hand side is proportional to $1-n$, and we come to the result consistent with (4.2). Thus, the decoupling of the Wick theorem type leads for the second derivative to the same result as on the basis of representation (3.1).

Consider now the third order derivative

$$
\frac{1}{\mathcal{Z}} \frac{\delta^{3} \mathcal{Z}}{\delta t_{12}^{\sigma} \delta t_{34}^{\bar{\sigma}} \delta t_{56}^{\bar{\sigma}}}=\left\langle\hat{\mathrm{T}} \psi_{\sigma}(1) \psi_{\sigma}^{\dagger}(2) \psi_{\bar{\sigma}}(3) \psi_{\bar{\sigma}}^{\dagger}(4) \psi_{\bar{\sigma}}(5) \psi_{\bar{\sigma}}^{\dagger}(6)\right\rangle
$$

Let us consider a system of pairing, when only $\psi$ and $\psi^{\dagger}$ operators are paired, for example,

$$
\begin{array}{r}
\langle\hat{\mathrm{T}} \psi_{\sigma}(1) \underbrace{\dagger}_{\sigma}(2) \psi_{\bar{\sigma}}(3) \psi_{\bar{\sigma}}^{\dagger}(4) \psi_{\overline{\bar{\sigma}}}(5) \psi_{\bar{\sigma}}^{\dagger}(6)\rangle=G_{\sigma}(14) G_{\bar{\sigma}}(32) G_{\bar{\sigma}}(56)\left\langle\hat{\mathrm{T}} X_{2}^{\sigma \bar{\sigma}} X_{4}^{\bar{\sigma} \sigma} F_{6}^{\bar{\sigma}}\right\rangle \\
=-G_{\sigma}(14) G_{\bar{\sigma}}(32) G_{\bar{\sigma}}(56)\left[\frac{1}{\mathcal{Z}} \frac{\delta^{3} \mathcal{Z}}{\delta t_{24}^{\bar{\sigma}} \delta t_{42}^{\sigma} \delta t_{66}^{\sigma}}-\mathcal{D}_{1}^{\sigma}(24)\right] .
\end{array}
$$

Taking only this system of pairing in (5.6) we have approximately

$$
\frac{1}{\mathcal{Z}} \frac{\delta^{3} \mathcal{Z}}{\delta t_{12}^{\sigma} \delta t_{34}^{\bar{\sigma}} \delta t_{56}^{\bar{\sigma}}} \approx-G_{\sigma}(14) G_{\bar{\sigma}}(32) G_{\bar{\sigma}}(56)\left[\frac{1}{\mathcal{Z}} \frac{\delta^{3} \mathcal{Z}}{\delta t_{24}^{\bar{\sigma}} \delta t_{42}^{\sigma} \delta t_{66}^{\sigma}}-\mathcal{D}_{1}^{\sigma}(24)\right]+\text { Symm. }
$$

Putting arguments $4=1,3=2,5=6$ we get an equation for a six-tailed diagram with equal arguments. For the paramagnetic phase this equation is as follows:

$$
\left[1+\left(\frac{n / 2}{1-n / 2}\right)^{3}\right] \frac{1}{\mathcal{Z}} \frac{\delta^{3} \mathcal{Z}}{\delta t_{24}^{\bar{\sigma}} \delta t_{42}^{\sigma} \delta t_{66}^{\sigma}} \approx\left(\frac{n / 2}{1-n / 2}\right)^{3} \mathcal{D}_{1}^{\sigma}(24) .
$$

This third order derivative with equal arguments corresponds to a six-tailed diagram with joint lines. Comparing relation (5.8) with the graphs in figure 5 shows that decoupling by the Wick theorem corresponds to the two graphs explicitly shown in figure 5 . One of them is a flower with three clovers. As we see from equation (5.9), the flower does not have a singular factor $1-n$.

A different situation occurs if the forth order derivative (eigt-tailed) is studied by the same method. When calculating T-product of eight $\psi$-operators, we take into account only two systems of pairing:

$$
\begin{aligned}
& \left\langle\hat{\mathrm{T}} \psi_{\sigma}(1) \psi_{\sigma}^{\dagger}(2) \psi_{\bar{\sigma}}(3) \psi_{\overline{\bar{\sigma}}}^{\dagger}(4) \psi_{\sigma}(5) \psi_{\sigma}^{\dagger}(6) \psi_{\bar{\sigma}}(7) \psi_{\bar{\sigma}}^{\dagger}(8)\right\rangle= \\
& =G_{\sigma}(14) G_{\bar{\sigma}}(32) G_{\sigma}(58) G_{\bar{\sigma}}(76) \mathcal{D}_{1}^{\sigma}(24) \mathcal{D}_{1}^{\sigma}(68)\left\langle F_{4}^{\sigma}\right\rangle\left\langle F_{8}^{\sigma}\right\rangle+ \\
& \quad+G_{\sigma}(14) G_{\bar{\sigma}}(32) G_{\sigma}(58) G_{\bar{\sigma}}(76)\left\langle\hat{\mathrm{T}} X_{2}^{\sigma \bar{\sigma}} X_{4}^{\bar{\sigma} \sigma} X_{6}^{\sigma \bar{\sigma}} X_{8}^{\bar{\sigma} \sigma}\right\rangle+\ldots
\end{aligned}
$$




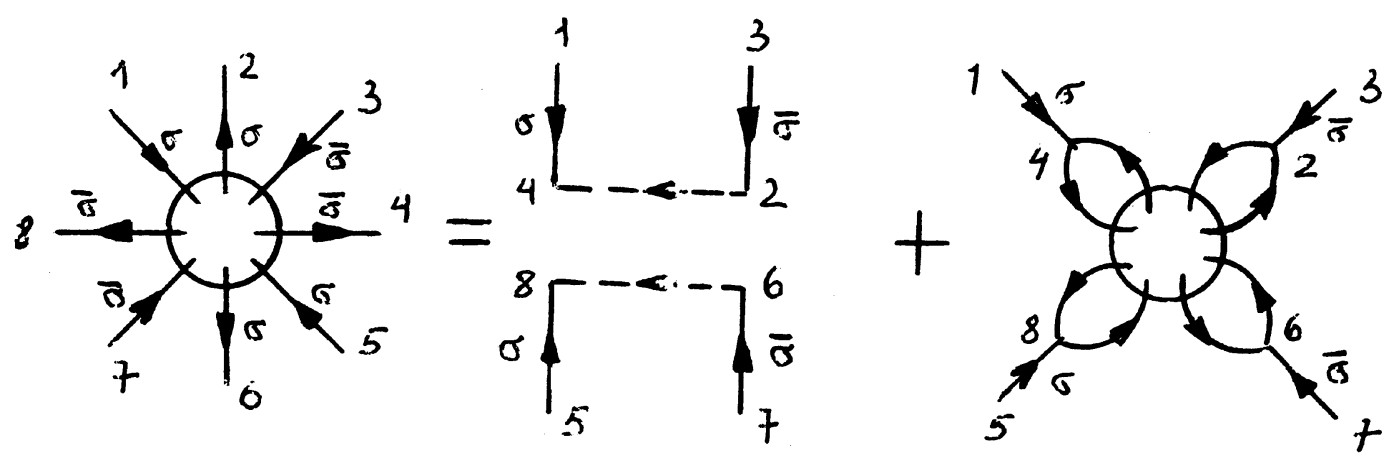

Figure 7. The forth order variational derivative in zero approximation.

Graphic expressions of these terms are shown in figure 7. The average of T-product of four b-operators corresponds to the fourth order derivative with coinciding arguments in pairs. Putting in (5.10) the corresponding arguments equal, one obtains an equation:

$$
\left[1-\left(\frac{n / 2}{1-n / 2}\right)^{4}\right] \frac{1}{\mathcal{Z}} \frac{\delta^{4} \mathcal{Z}}{\delta t_{42}^{\sigma} \delta t_{24}^{\bar{\sigma}} \delta t_{86}^{\sigma} \delta t_{68}^{\bar{\sigma}}}=\left(\frac{n / 2}{1-n / 2}\right)^{4} \mathcal{D}_{1}^{\sigma}(24) \mathcal{D}_{1}^{\sigma}(68)\left\langle F_{4}^{\sigma}\right\rangle\left\langle F_{8}^{\sigma}\right\rangle .
$$

We see from here that the derivative has a singular factor $1-n$ in the denominator. The difference in the third and fourth derivatives is caused by different signs in diagrams of the flower type in six-tailed and eight-tailed diagrams. It is easy to

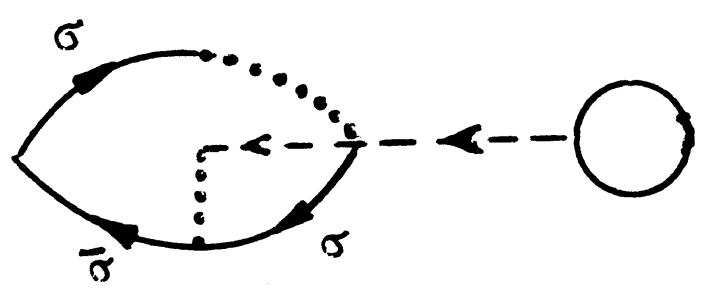

Figure 8. A second order correction to magnon Green function. see that the singular factor should always appear in the flower type diagrams with an even number of clovers.

If in expression (5.10) for the fourth derivative we take only the last term, we obtain a second order correction in the magnon Green function presented in figure 8. For this graph an analytical contribution into the denominator of expression (4.3) is

$$
\frac{1}{8(1-n)} \sum_{\mathbf{k}} J(\mathbf{k}-\mathbf{q}) G_{\sigma}(\mathbf{k}-\mathbf{q}) G_{\bar{\sigma}}(\mathbf{k})\left(\sum_{\mathbf{q}_{1}} J\left(\mathbf{q}_{1}\right) \mathcal{D}_{1}^{\sigma}\left(\mathbf{q}_{1}\right) G_{\sigma}\left(\mathbf{k}-\mathbf{q}_{1}\right)\right) .
$$

Of course, a symmetrical graph in figure 8 should be added which gives expression (5.12) with the change $\mathbf{q} \rightarrow-\mathbf{q}$.

Due to singular factor $1-n$, the second order corrections are as important, when $n \rightarrow 1$, as the first order ones. Zero in the denominator of the magnon Green function (4.3) at $\omega=0$ and $\mathbf{q}=\mathbf{Q}$ determines a boundary of the paramagnetic phase stability with respect to the appearance of an antiferromagnetic order. As one can 
see from $(4.3)$ and $(5,12)$, this boundary goes along the line of type $1-n \sim J / t$, as it should be. Detailed analysis of the magnetic phase diagram will be given elsewhere.

\section{Conclusions}

We have generalized the Kadanoff-Baym approach to systems with strong electron correlations and applied it to a $t J$-model. The $X$-operator representation allows one to derive equations for variational derivatives of the generating functional in a form convenient for iteration with respect to $t$ and $J$. Within the framework of the general approach we suggested an approximation of the SCBA type, where electron and boson Green functions are considered as exact, and vertex parts are expanded over $t$ and $J$. In such an approach the problem of calculation of the Green functions reduces to the calculation of the variational derivatives in the "zero" approximation with exact Green functions. It opens a possibility to obtain self-consistent equations for Green functions.

We have suggested a method for the calculation of variational derivatives in the zero approximation, which gives a correct symmetry arising from the possibility to change the order of differentiation. The results for such calculations are too cumbersome and we presented them only for the second derivatives. It is remarkable that they are consistent with the diagram technique for $X$-operators based on the generalized Wick theorem [7].

We used this formalism for the calculation of the magnon Green function in the paramagnetic phase. To avoid cumbersome calculations of higher order derivatives based on the representation of the (3.1)-type we calculated the third order derivative by using the Wick theorem, because in this case the exact symmetry is not important. We have found graphs giving a growing contribution when the system tends to half-filling. In $n \rightarrow 1$ limit only graphs of the second order containing singular factor $(1-n)^{-1}$ should be taken into account. They produce instability of the paramagnetic phase with respect to antiferromagnetic ordering. Singular contribution (5.12) in the denominator of the magnon Green function comes from the graph in figure 8. One can see that it involves a magnon in the intermediate state. The corresponding physical process is the following: a magnon creates an electron-hole pair with the same spins and still it exists itself. Then, one of particles of the pair absorbs this magnon. As a final result, an electron-hole pair with the opposite spins appears. Notice that the possibility of such a virtual process is connected with the existence of two types of vertices for the electron-magnon interaction in the diagram technique for the $t J$-model: elastic and inelastic ones. Both vertices are involved in the second order graph.

We consider the results of the calculation of the magnon Green function as preliminary ones. It is necessary to look for singular terms among high order diagrams. It is also necessary to calculate the plasmon Green functions (2.10), particularly for the longitudinal spin deviations, and also to calculate the second order correction to the electron Green function. In this way a self-consistent system 
of equations for boson and electron Green functions can be obtained.

This work was supported by the State Science and Technology Committee, the program Superconductivity, grant No 95-056.

\title{
References
}

1. Izyumov Yu.A. The $t J$-model for strongly correlated electrons and high- $T_{\mathrm{c}}$-superconductors. // Uspekhi Fiz. Nauk, 1997, vol. 167, No 5, p. 465-497 (in Russian).

2. Kadanoff L., Baym G. Quantum statistical mechanics: Green's function methods in equilibrium and nonequilibrium problems. New-York: Benjamin, 1962.

3. Izyumov Yu.A., Chashchin N.I. Selfconsistent interaction of quasiparticles with fluctuations of magnetic order in the $t J$-model. // Fiz. Met. Metallography, 1998, vol. 85, No 3, p. 5-19 (in Russian).

4. Plakida N.M., Yushankhai V.Yu., Stasyuk I.V. On the role of kinematic and exchange interactions in superconducting pairing of electrons in the Hubbard model. // Physica C, 1989, vol. 160, p. 80-88.

5. Slobodyan P.M., Stasyuk I.V. Diagram technique for Hubbard operators. // Teor. Mat. Fiz., 1974, vol. 19, No 3, p. 423-431 (in Russian).

6. Izyumov Yu.A., Katsnelson M.I., Scryabin Yu.N. Magnetism of itinerant electrons. Moscow, Fiziko-Matematicheskaya Literatura, 1994, 366 p.

7. Izyumov Yu.A., Letfulov B.M. A diagram technique for Hubbard operators: the magnetic phase diagram in the $t J$-model. // J. Phys.: Cond. Matt., 1990, vol. 2, No 45, p. 8905-8923.

\section{$t J$-модель в термінах рівнянь з варіаційними похідними}

\author{
Ю.О.Ізюмов, Н.І.Чащін
}

Уральське відділення Інституту металофізики РАН, Росія, 620219 Єкатєрінбург, вул. С.Ковалевської, 18

Отримано 17 червня 1998 р.

Для $t J$ моделі в представленні $X$-операторів введено узагальнений функціонал поля, що описує флуктуації матричних елементів електронних перескоків на гратці. Функціональна похідна першого порядку по цьому полю визначає електронну функцію Гріна, тоді як похідні другого порядку визначають бозонні функції Гріна колективних збуджень в системі. Таким чином узагальнено підхід КадановаБейма в теорії фермі-систем з слабою кулонівською взаємодією на протилежний випадок систем з сильними кореляціями. Отримано ланцюжок рівнянь для варіаційних похідних різного порядку і запропоновано метод, що базується на ітераціях за параметрами $t J$ моделі: матричним елементом перескоку і обмінним інтегралом. Цей підхід відповідає самоузгодженому наближенню Борна, але не для 
ефективного, а для вихідного гамільтоніану. Аналізується схема розрахунку динамічної спінової сприйнятливості з самоузгодженими поправками першого і другого порядку. Обговорюється зв'язок цього підходу з діаграмною технікою для $X$-операторів.

Ключові слова: $t J$-модель, оператори Хаббарда, функції Гріна, антиферомагнетизм

PACS: $71.27 .+a, 74.90 .+n$ 